\title{
Exploring effects of remote ischemic preconditioning in a pig model of hypothermic circulatory arrest
}

\section{Herajarvi, Johanna}

2017

Herajarvi , J , Anttila , T , Dimova , E Y , Laukka , T, Myllymaki , M , Haapanen , H , Olenchock, B A, Tuominen , H , Puistola , U , Karihtala , P , Kiviluoma , K, Koivunen , P , Anttila , V \& Juvonen , T 2017 , ' Exploring effects of remote ischemic preconditioning in a pig model of hypothermic circulatory arrest ' , Scandinavian Cardiovascular Journal , vol. 51, no. 4 , pp. 233-241 . https://doi.org/10.1080/14017431.2017.1319574

http://hdl.handle.net/10138/237095

https://doi.org/10.1080/14017431.2017.1319574

unspecified

publishedVersion

Downloaded from Helda, University of Helsinki institutional repository.

This is an electronic reprint of the original article.

This reprint may differ from the original in pagination and typographic detail.

Please cite the original version. 


\section{Scandinavian Cardiovascular Journal}

\section{Exploring effects of remote ischemic preconditioning in a pig model of hypothermic circulatory arrest}

Johanna Herajärvi, Tuomas Anttila, Elitsa Y. Dimova, Tuomas Laukka, Mikko Myllymäki, Henri Haapanen, Benjamin A. Olenchock, Hannu Tuominen, Ulla Puistola, Peeter Karihtala, Kai Kiviluoma, Peppi Koivunen, Vesa Anttila \& Tatu Juvonen

To cite this article: Johanna Herajärvi, Tuomas Anttila, Elitsa Y. Dimova, Tuomas Laukka, Mikko Myllymäki, Henri Haapanen, Benjamin A. Olenchock, Hannu Tuominen, Ulla Puistola, Peeter Karihtala, Kai Kiviluoma, Peppi Koivunen, Vesa Anttila \& Tatu Juvonen (2017) Exploring effects of remote ischemic preconditioning in a pig model of hypothermic circulatory arrest, Scandinavian Cardiovascular Journal, 51:4, 233-241, DOI: 10.1080/14017431.2017.1319574

To link to this article: http://dx.doi.org/10.1080/14017431.2017.1319574

+ View supplementary material

Submit your article to this journal
Published online: 24 Apr 2017.

Џll Article views: 68

View Crossmark data $\nearrow$ 


\title{
Exploring effects of remote ischemic preconditioning in a pig model of hypothermic circulatory arrest
}

\author{
Johanna Herajärvi ${ }^{\mathrm{a}}$, Tuomas Anttila ${ }^{\mathrm{a}}$, Elitsa Y. Dimova ${ }^{\mathrm{b}}$, Tuomas Laukka ${ }^{\mathrm{b}}$, Mikko Myllymäki $^{\mathrm{b}}$, Henri Haapanen ${ }^{\mathrm{a}}$, \\ Benjamin A. Olenchock ${ }^{c}$, Hannu Tuominen ${ }^{d}$, Ulla Puistola ${ }^{e}$, Peeter Karihtala ${ }^{f}$, Kai Kiviluoma ${ }^{a}$, Peppi Koivunen ${ }^{b}$, \\ Vesa Anttila ${ }^{\mathrm{a}, \mathrm{g}}$ and Tatu Juvonen ${ }^{\mathrm{a}, \mathrm{h}}$

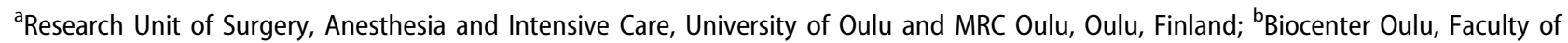 \\ Biochemistry and Molecular Medicine, Oulu Center for Cell-Matrix Research, University of Oulu, Oulu, Finland; 'Division of Cardiovascular \\ Medicine, Department of Medicine, The Brigham and Women's Hospital, Harvard Medical School, Boston, MA, USA; ${ }^{\mathrm{d} D e p a r t m e n t}$ of

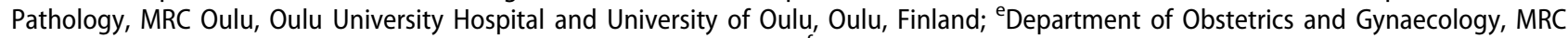 \\ Oulu, Oulu University Hospital and University of Oulu, Oulu, Finland; ' Department of Oncology and Radiotherapy, MRC Oulu, Oulu University \\ Hospital and University of Oulu, Oulu, Finland; ${ }^{9}$ Heart Center, Turku University Hospital, University of Turku, Turku, Finland; ${ }^{h}$ Department of \\ Cardiac Surgery, HUCH Heart and Lung Center, Helsinki, Finland
}

\begin{abstract}
Objectives. During aortic and cardiac surgery, risks for mortality and morbidity are inevitable. Surgical setups involving deep hypothermic circulatory arrest (DHCA) are effective to achieve organ protection against ischemic injury. The aim of this study was to identify humoural factors mediating additive protective effects of remote ischemic preconditioning (RIPC) in a porcine model of DHCA. Design. Twentytwo pigs were randomized into the RIPC group $(n=11)$ and the control group $(n=11)$. The RIPC group underwent four 5-minute hind limb ischemia-reperfusion cycles prior to cardiopulmonary bypass and DHCA. All animals underwent identical surgical procedures including $60 \mathrm{~min}$ DHCA at $18^{\circ} \mathrm{C}$. Blood samples were collected from vena cava and sagittal sinus at several time points. After the 8-hour follow-up period, the brain, heart, and kidney tissue samples were collected for tissue analyses. Results. Serum levels of brain damage marker S100B recovered faster in the RIPC group, after 4 hours of the arrest, $(p<.05)$. Systemic lactate levels were lower and cardiac index was higher in the RIPC group postoperatively. Immunohistochemical cerebellum regional scores of antioxidant response regulator Nrf2 were better in the RIPC group (mean: 1.1, IQR: 0.0-2.5) compared with the control group (mean: 0.0, IQR: $0.0-0.0)$, reaching borderline statistical significance $(p=.064)$. RIPC induced detectable modulations of plasma proteome and metabolites. Conclusions. The faster recovery of S100B, lower systemic lactate levels and favourable regional antioxidant response suggest possible neuronal cellular and mitochondrial protection by RIPC, whereas better cardiac index underlines functional effects of RIPC. The exact humoural factor remains unclear.
\end{abstract}

\section{ARTICLE HISTORY}

Received 6 November 2016

Revised 10 April 2017

Accepted 11 April 2017

\section{KEYWORDS}

Remote ischemic preconditioning; hypothermic circulatory arrest; humoural factors

\section{Introduction}

Deep hypothermic circulatory arrest (DHCA) is a widely used operative technique in the surgical procedures of aortic pathologies requiring the cessation of the blood supply. For instance, this method is used in the surgical repair of acute type A aortic dissection, congenital heart defects, and thoracoabdominal aortic aneurysms [1-3]. The cerebral protection of this technique is based on the suppression of the central nervous system (CNS) metabolic demand increasing neuronal ischemic tolerance $[4,5]$. In spite of the hypothermic neuroprotection, postoperative mortality of $2.4 \%$ to $6.3 \%$ and a neurological deficit of $1.6 \%$ to $4.8 \%$ still exist in reported series using DHCA, establishing adjunctive protective strategies to be studied $[2,6]$.

Ischemic preconditioning was first introduced in a canine model, with an intermittent occlusion of the left circumflex artery (LCX) prior to prolonged occlusion of LCX, limiting myocardial infarct size [7]. This preconditioning method, exposing target tissue to an ischemic stimulus providing protection against subsequent more severe insult, was later found to be similarly effective when applied at a distance along with another nontarget tissue $[8,9]$. Consequently, cardio- and neuroprotective effects of remote ischemic preconditioning (RIPC) have been widely studied both in experimental and in clinical settings [10-14].

The protective effects of RIPC are well documented among different mammalian species; however, the underlying molecular mechanisms involved in this protection remain largely unclear. At present, there are three theories: the humoural, the neural, and the systemic [15]. The largest body of preclinical data supports the concept of an RIPCinduced factor(s) in the bloodstream that conveys protection

CONTACT Johanna Herajärvi Johanna.herajarvi@oulu.fi @ Research Unit of Surgery, Anesthesia and Intensive Care, University of Oulu and MRC Oulu, Finland P.O. Box 21, 90029 OYS, Oulu, Finland

(1) Supplemental data for this article can be accessed here.

(C) 2017 Informa UK Limited, trading as Taylor \& Francis Group 

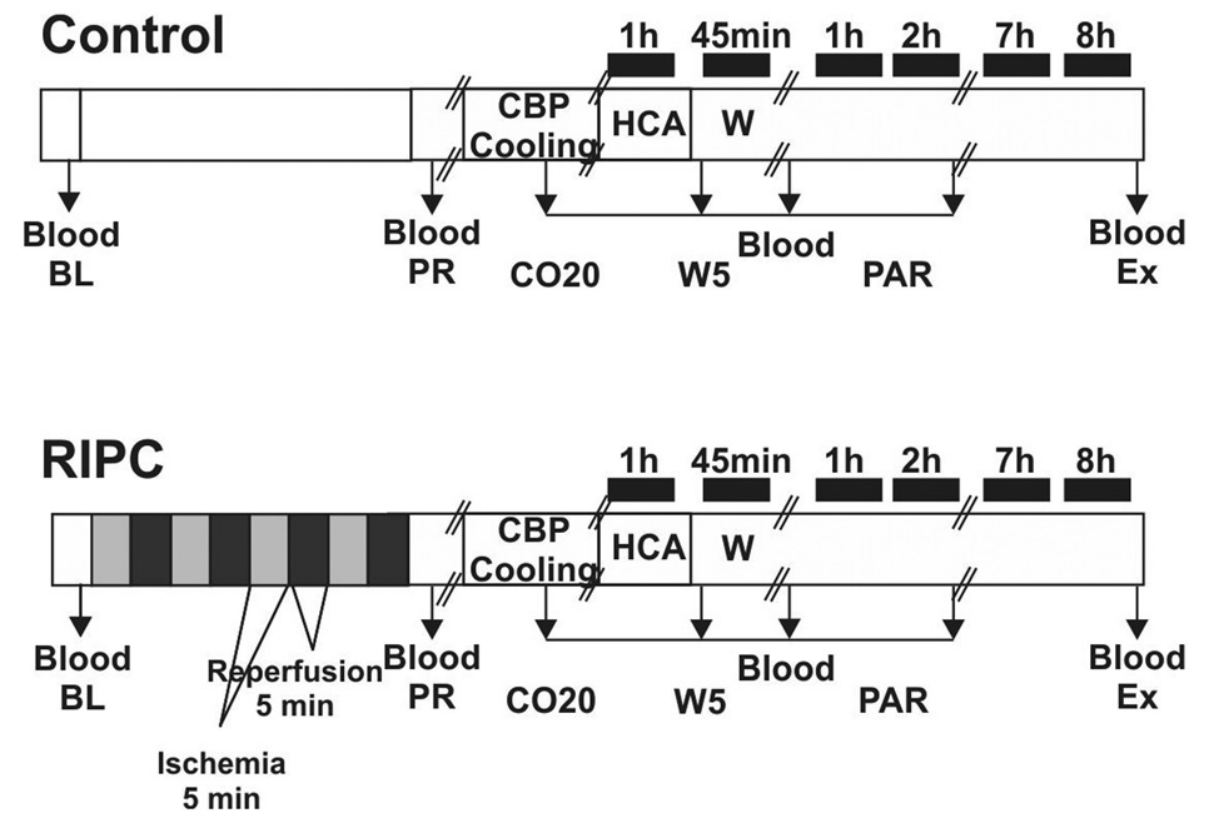

Figure 1. Experimental setup with samplings. BL: baseline time point; CBP: cardiopulmonary bypass; CO20: cooling 20 minutes time point; EX: exitus time point; HCA: hypothermic circulatory arrest; PAR: postarrest time period; PR: postintervention time point; RIPC: remote ischemic preconditioning; W: warming time period; W5: rewarming phase after arrest time point.

from the transiently ischemic limb to the remote organ(s) [16-22]. Moreover, the preconditioning protection has been shown to be transferable with the plasma, even in cases of cross-species transfer $[17,18,23,24]$ and it appeared to be mediated via a small, unknown hydrophobic factor under $30 \mathrm{kDa}[17,25]$.

Oxidative stress, imbalance between the production of reactive oxygen species (ROS) and antioxidant defence system, and especially restricted production of ROS are considered one of the possible mechanisms behind the preconditioning phenomenon [26]. One of the key antioxidant response regulators, nuclear factor erythroid 2-related factor, Nrf2, senses the cellular redox status [27]. Ischemiareperfusion stimulus, low production of ROS, and consequently mild oxidative stress, induce the translocation of Nrf2 from cytoplasm to nucleus, leading to the induction of various antioxidant enzymes.

Moreover, it was recently reported that preconditioning via inhibition of EGLN1, a hypoxia-inducible factor (HIF) prolyl 4-hydroxylase, in skeletal muscle reroutes $\alpha$-ketoglutarate $(\alpha \mathrm{KG})$, which drives hepatic production and secretion of kynurenic acid (KYNA) that is necessary and sufficient to mediate cardiac ischemic protection in mice [28].

The objective of the study was to assess the additive protective effects of RIPC and identify different humoural circulating factors mediating the mechanisms of RIPC in a porcine model of DHCA.

\section{Materials and methods}

\section{Experimental setup}

Twenty-two female pigs from a native stock (aged 7-8 weeks) were randomized into two groups, a control group $(\mathrm{n}=11)$ and a remote ischemic preconditioning (RIPC) group $(\mathrm{n}=11)$ using sealed envelopes. All animals underwent 60 minutes of deep hypothermic circulatory arrest at $18{ }^{\circ} \mathrm{C}$. In advance of cardiopulmonary bypass $(\mathrm{CPB})$ procedures, the RIPC group underwent 4 cycles of 5-minute right hind limb ischemia-reperfusion episodes. Schematic representation of the experimental setup and sampling is shown in the Figure 1.

All animals received humane care in accordance with the Principles of Laboratory Animal Care formulated by the National Society for Medical Research and the Guide for the Care and Use of Laboratory Animals prepared by the Institute of Laboratory Animal Resources, National Resource Council (Published by National Academy Press, revised 1996). The study protocol was approved by the Research Animal Care and Use Committee of the University of Oulu.

\section{Anesthesia and cardiopulmonary bypass protocols}

The anesthesia, hemodynamic and cardiopulmonary bypass protocols are described in detail in the online version, Supplementary Material.

\section{Cranial procedures}

A cranial window $(35 \times 35 \mathrm{~mm})$ was extracted on top of the scalp using a 14/11 mm disposable cranial perforator (200-253 DGR-II, Acra Cut Inc, Acton MA). A peripheral venous cannula $\left(22 \mathrm{G} \times 25 \mathrm{~mm}\right.$ BD Venflon ${ }^{\mathrm{TM}}$ Peripheral IV Catheter with Injection port) for blood sampling was inserted through dura mater to the sagittal sinus and secured thoroughly.

\section{Remote ischemic preconditioning (RIPC)}

In remote ischemic preconditioning, a children's blood pressure cuff was placed around the right hind limb. The cuff 
was inflated to $>250 \mathrm{mmHg}$ for 5 minutes followed by a 5 minute deflation. This intermittent ischemia-reperfusion cycle was repeated four times, and the total time of RIPC stimulus lasted for 40 minutes, as in previous studies $[7,10]$. In the control group the blood pressure cuff was placed for 40 minutes around the right hind limd without any inflation-deflation cycles. Preconditioning was performed 65 minutes (IQR: $51-72$ minutes, $\mathrm{p}=.752$ ) prior to $\mathrm{CPB}$, and hypothermic circulatory arrest was achieved 95 minutes from the last preconditioning cycle targeting for the first protective window of RIPC [29].

\section{Postoperative management}

Intravenous noradrenalin infusion was used postoperatively if necessary to maintain mean arterial pressure over $60 \mathrm{mmHg}$. Animals were under terminal anesthesia, which together with mechanical ventilation were maintained for 8 hours after DHCA followed by euthanization using pentobarbital $(90 \mathrm{mg} / \mathrm{kg})$.

\section{Sample collection and biochemical data}

Cardiac output, pulmonary capillary wedge pressure, urine volume, infused fluid volume, and inotropes administered, as well as rectal and pulmonary artery temperature readings, were recorded. Blood count, cardiac troponin I (TnI), creatine kinase isoenzyme $\mathrm{MB}(\mathrm{CK}-\mathrm{MBm})$, blood gas values, $\mathrm{pH}$, electrolytes, serum ionized calcium, plasma lactate levels, venous glucose, hematocrit, and hemoglobin levels (iSTAT Analyzer; iSTAT Corporation, East Windsor, NJ) were measured at baseline (BL), at the end of the remote ischemic preconditioning or sham treatment $(\mathrm{PR})$, after 20 minutes of cooling (CO20), at 5 minutes (W5), and 1, 2, 4, 8 hours from the end of the DHCA.

\section{Collection of blood and isolation of serum samples from vena cava and sagittal sinus}

Blood samples were collected from two different vessels, vena cava (VC) and sagittal sinus (SS), at the aforementioned time points. The proteomics analyses were carried out in the Proteomics core facility at Karolinska Institute, Stockholm, Sweden. The exact preparation of protein extraction and solubilization, in solution digestion, PRLC-MS/MS analysis, data processing, protein quantitation and ELISA are described in detail in the online version, Supplementary Material.

\section{Protein biomarker for brain damage}

Serum S100B levels were analysed with ELISA kit (Fujirebio Diagnostics) following the manufacturer's instructions.

\section{Analysis of KYNA and aKG levels}

The KYNA and $\alpha \mathrm{KG}$ levels in serum were analyzed as in Olenchock et al. 2016 [28].

\section{Histopathological analysis and immunohistochemistry}

After euthanization, the tissue samples were collected from the brain, the right ventricle of the heart and the right kidney and fixed in $10 \%$ formalin solution. The preparation methods and scoring systems of histopathological and immunohistochemical analyses are described in the online version, Supplementary Material.

\section{Statistical analysis}

SPSS (version 22.0; SPSS Inc, Chicago, IL) and SAS (version 9.3; SAS Institute, Cary, NC) statistical software packages were used for statistical analysis. Continuous and ordinal variables are expressed as the median with interquartile range (IQR; 25th and 75th percentiles). In the figures, mean and standard error of mean (SEM) are used. Complete independence was assumed across all animals (by random statement). Distribution of the variable was tested using the Shapiro-Wilks test of normality. Either the Student $t$ test or Mann-Whitney $U$ test was used to assess the $p$ values between the variables of the study groups. Two-tailed significance levels are reported. $\mathrm{p} \leq .05$ was considered statistically significant. In the proteomics analysis of serum samples, non-parametric statistic tests, Friedman and Dunn's multiple comparison tests, were used to detect differences across multiple sample populations.

The repeatedly measured data was analyzed using a linear mixed model with animals fitted as random, and the covariance pattern was chosen according to Akaike's Information Criterion. Reported $\mathrm{p}$ values are as follows: $\mathrm{p}$ between groups $\left(p_{g}\right)$ indicates a level of difference between the groups; $\mathrm{p}$ for time by group $\left(p_{t^{*} g}\right)$ indicates behaviour between the groups with time.

\section{Results}

\section{Mortality}

A total of 22 animals were operated on. Six animals were excluded from the analyses. Four animals were lost during the sagittal sinus or aortic cannulations. One animal from each study group was lost during the follow-up period due to acute heart failure. Eventually, 16 animals were included in the final analysis.

\section{Comparability of study groups}

The mean weight of the pigs in the control group was $19.1 \mathrm{~kg}$ and in the RIPC group $20.0 \mathrm{~kg}(\mathrm{p}=.316)$. The amount of transfused blood was $69.0 \mathrm{ml} / \mathrm{kg}(62.5-78.6 \mathrm{ml} / \mathrm{kg})$ in the intervention group and $68.7 \mathrm{ml} / \mathrm{kg}(62.3-74.4 \mathrm{ml} / \mathrm{kg})$ in the control group, respectively $(\mathrm{p}=.767)$.

\section{Experimental and metabolic data}

Systemic concentration of venous lactate was lower in the intervention group after the operation, and the baseline level was more rapidly reached in the RIPC group (Figure 2(A)). 

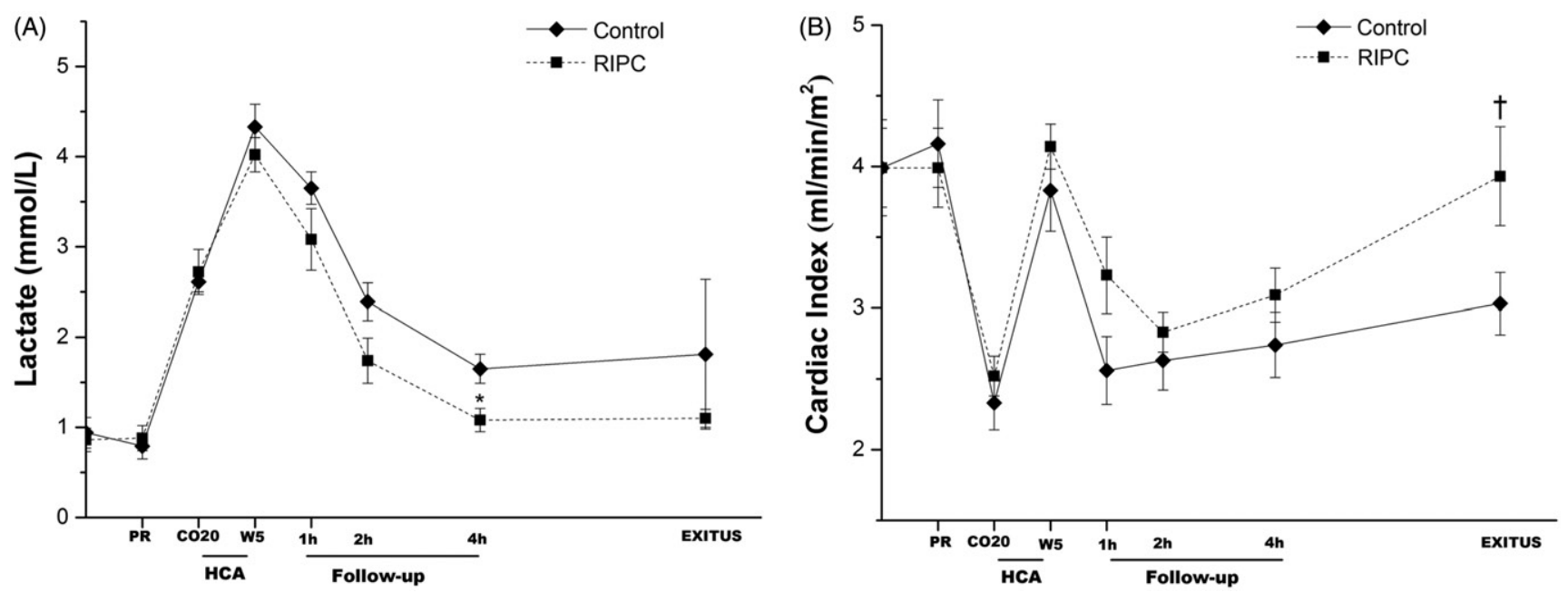

Figure 2. Systemic concentration of lactate (2A). Cardiac index (2B). CO20: cooling 20 minutes time point; HCA: hypothermic circulatory arrest; PR: postintervention time point; RIPC: remote ischemic preconditioning; W5: rewarming phase after arrest time point. Mean \pm SEM is presented. ${ }^{*} p<.05,+p<.06$ at specific time point. Mann-Whitney $U$ test.

After weaning from the CPB, the RIPC group also demonstrated a better cardiac index reaching the baseline values (Figure 2(B)). The study groups did not differ in number of defibrillations $(\mathrm{p}=.494)$, the usage of noradrenaline need for inotropes after the operation $(\mathrm{p}=.643)$, or fluid balance at the end of the follow-up $(p=.987)$. Experimental and metabolic data are summarized in the online version, Supplementary Tables 2(A, B).

\section{Neurological markers}

The serum levels of S100B protein changed in a similar manner during the surgery in both groups. S100B protein levels peaked after DHCA during the initial rewarming phase and slowly decreased to baseline during the follow-up period in the control group. However, the serum levels of S100B protein declined faster in the RIPC group, reaching a significant difference compared with the control group at 4 hours after the arrest (Figure 3).

\section{Proteomics analysis of serum samples}

In the online version, Supplementary Table 1 shows all serum samples analysed and identification of peptides and proteins per sample. The number of proteins detected trustworthy was relatively low in all samples. This could be due to using sera non-depleted from major proteins, as well as the limited number of identified porcine proteins in the public databases. The samples from VC and SS were compared to the baseline from the corresponding vessel, and change of $\geq 20 \%$ in abundance was set as a threshold. From all reliably detected proteins in samples, 57 (42\%) were known porcine proteins, while $78(58 \%)$ were uncharacterized (Supplementary Table 1). The identified changes in protein levels in the control and RIPC groups in the indicated time points is presented in Figure 4.

Next, we used an open-source bioinformatics gene analysis tool (PANTHER) to map the biological processes these proteins are involved in. The known proteins were related

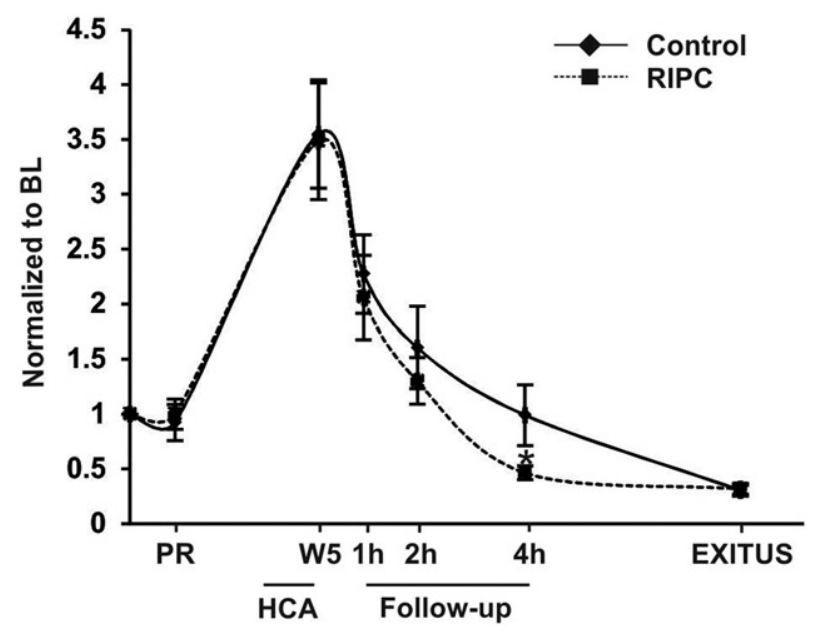

Figure 3. Serum concentration of $\mathrm{S} 100 \mathrm{~B}$ at different time points of the surgery. BL: baseline time point; HCA: hypothermic circulatory arrest; PR: postintervention time point; S100B: glial-specific S100 calcium binding protein B; W5: rewarming phase after arrest time point. Mean \pm SEM is presented. ${ }^{*} p<.05$ at specific time point. Student $t$ test.

to variety of biological processes such as metabolic, cellular, development, and immune system processes, and localization (Figure 5).

Particular interest was given to samples from sagittal sinus because they were taken behind the blood-brain barrier. Based on previous literature on protein functions and possible relevance to RIPC $[19,21,30]$ we selected 4 proteins:, apolipoprotein E (APOE), complement C3 (C3), transthyretin (TTR) and vitronectin (VTN), to be validated by ELISA. The levels of these four proteins were, however, not significantly changed between the RIPC and control groups at any of the studied time points (Figure 6).

\section{Analysis of serum KYNA and aKG levels}

The levels of $\alpha \mathrm{KG}$ in the vena cava samples decreased significantly following the first and third cycle of ischemiareperfusion with consistent oscillations in metabolite levels seen in all experimental animals (Figure 7) [28]. 
(A)

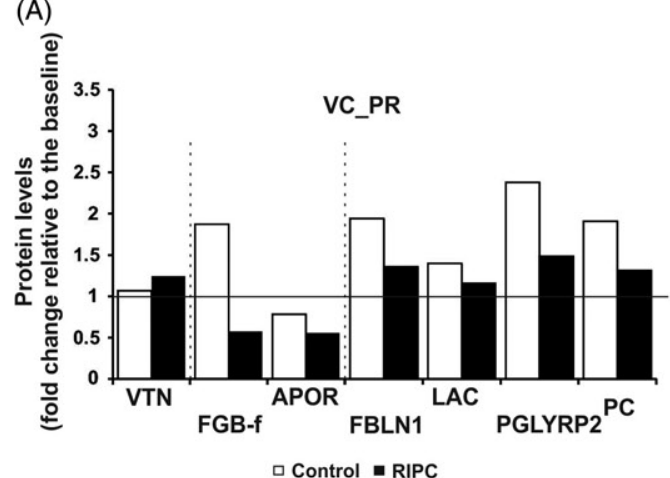

(C)

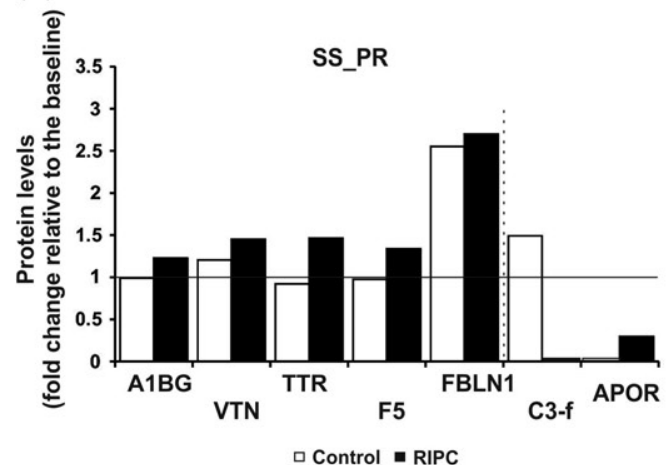

(B)

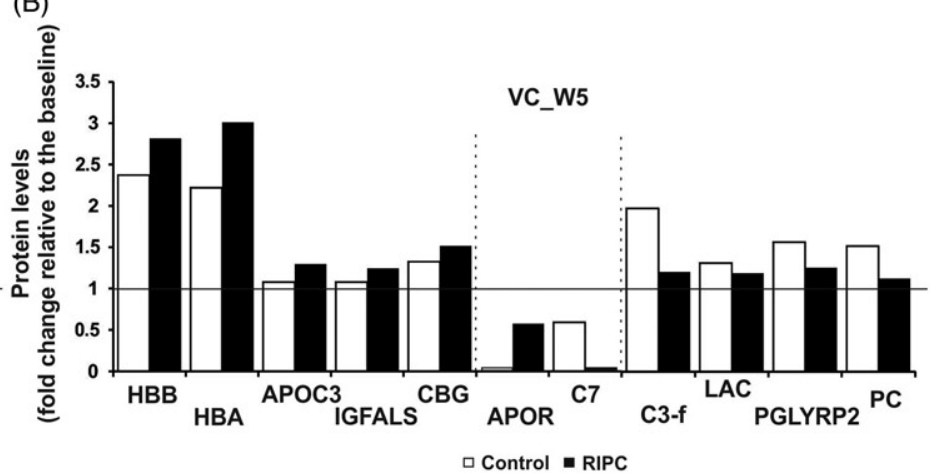

(D)

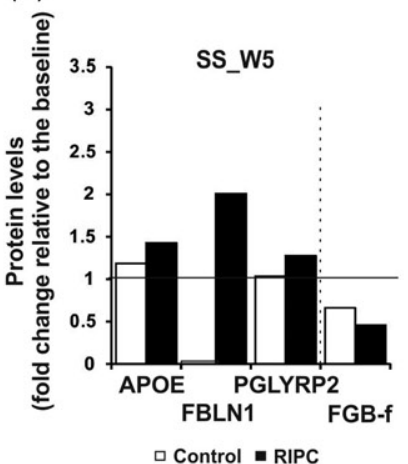

APOR - apolipoprotein R
VTN-vitronectin

FGB-f - fibrinogen $b$ chain (fragment)

APOR - apolipoprotein $R$

FBLN1 - fibulin-1

LAC - Ig lambda chain C region

PGLYRP2 - Isoform A of $\mathrm{N}$-acetylmuramoyl-L-alanine amidase

PC - Vitamin K-dependent protein C

HBB -hemoglobin subunit beta

HBA -hemoglobin subunit alpha

APOC3 - apolipoprotein C-III

IGFALS - acid-labile subunit

CBG - corticosteroid-binding globulin

C7 - complement component C7

C3-f- complement component C3 (fragment)

A1BG-alpha-1B-glycoprotein

F5- coagulation factor $V$

APOE-apolipoprotein E

Figure 4. Changed recognized porcine proteins at different time points of the HCA surgery. (A) VC_PR vs BL, (B) VC_W5 vs BL, (C) SS_PR vs BL, (D) SS_W5 vs BL. For analysis of the difference in expression change of $>20 \%$ was set as a treshold. BL: baseline time point; HCA: hypothermic circulatory arrest; PR: postintervention time point; SS: sagittal sinus; VC: vena cava; W5: rewarming phase after arrest time point.
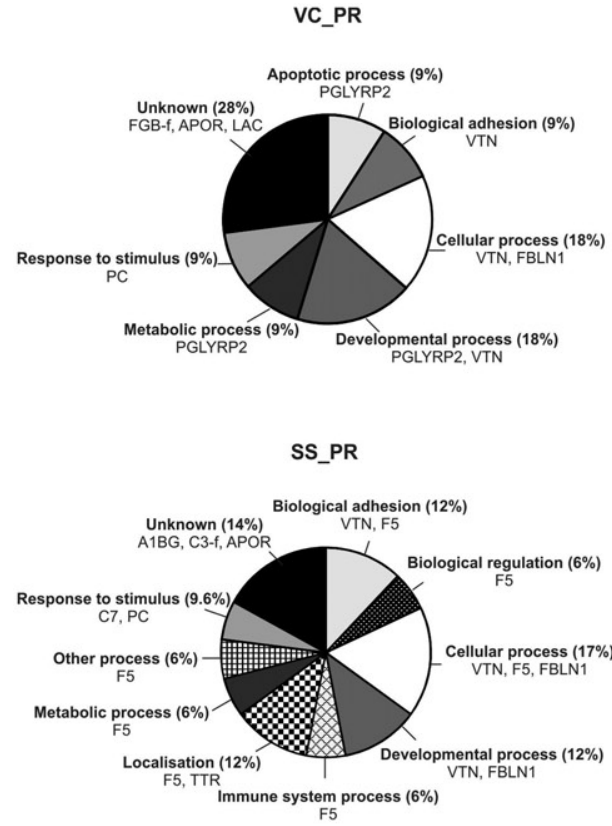

VC_W5

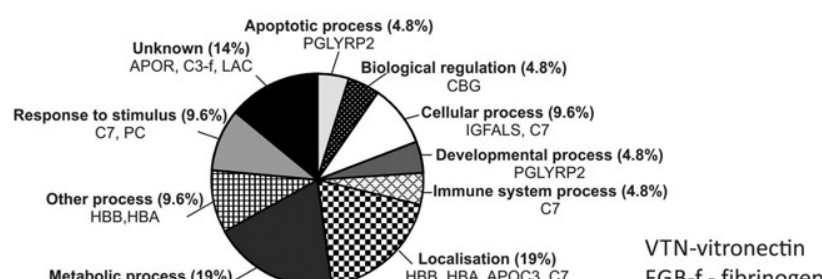

Metabolic process (19\%)
APOC3,CBG,C7,PGLYRP2 HBB, HBA, APOC $3, \mathrm{C} 7$

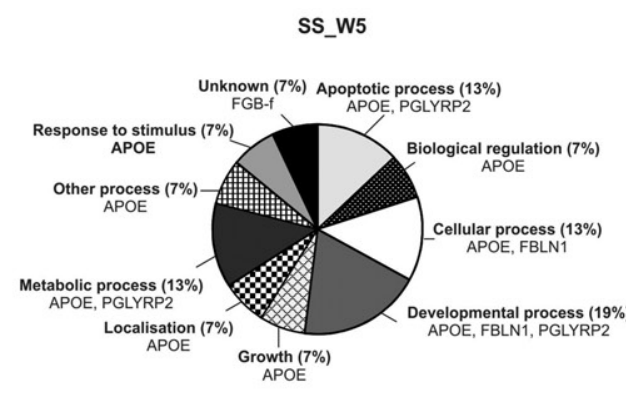

APOR - apolipoprotein R

FBLN1 - fibulin-1

LAC - Ig lambda chain C region

PGLYRP2 - Isoform A of

$\mathrm{N}$-acetylmuramoyl-L-alanine amidase

PC - Vitamin K-dependent protein C

HBB -hemoglobin subunit beta

HBA -hemoglobin subunit alpha

APOC3 - apolipoprotein C-III

IGFALS - acid-labile subunit

CBG - corticosteroid-binding globulin

APOR - apolipoprotein R

C7 - complement component C7

C3-f- complement component C3 (fragment)

A1BG- alpha-1B-glycoprotein

F5- coagulation factor $V$

APOE-apolipoprotein E

Figure 5. Pie chart representation of the biological functions of the known serum proteins using the PANTHER software. PR: postintervention time point; SS: sagittal sinus; VC: vena cava; W5: rewarming phase after arrest time point. 

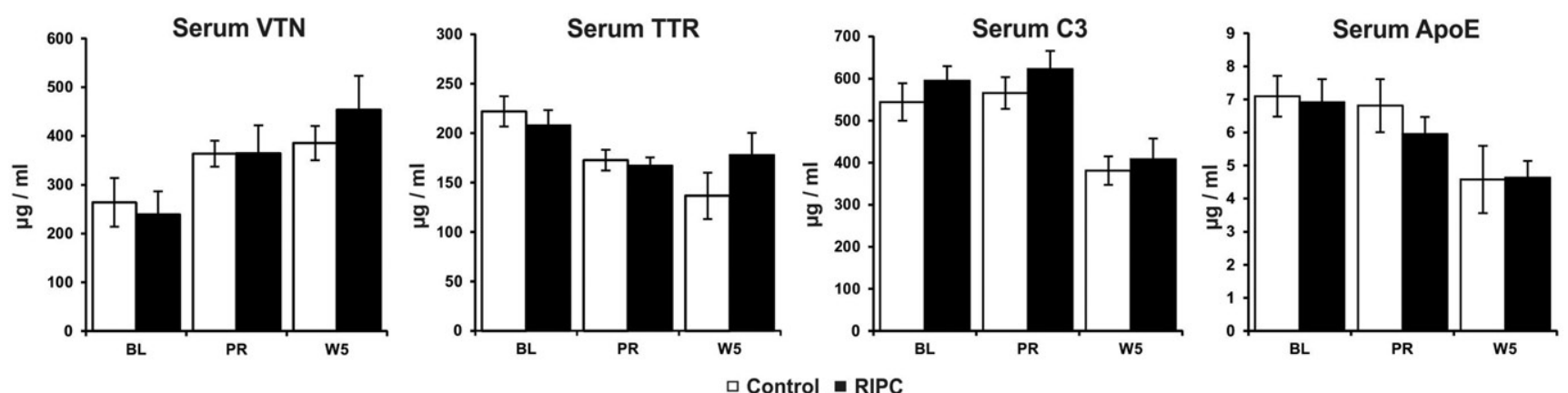

Figure 6. Sagittal sinus serum concentration of vitronectin (VTR), transthyretin (TTR), complement C3 (C3), and apolipoprotein E (ApoE) at baseline (BL), postintervention (PR), and rewarming phase after HCA (W5) in control (n: 8) and RIPC (n: 8) groups. BL: baseline time point; HCA: hypothermic circulatory arrest; PR: postintervention time point; RIPC: remote ischemic preconditioning; W5: rewarming phase after arrest time point. Mean \pm SEM is presented.

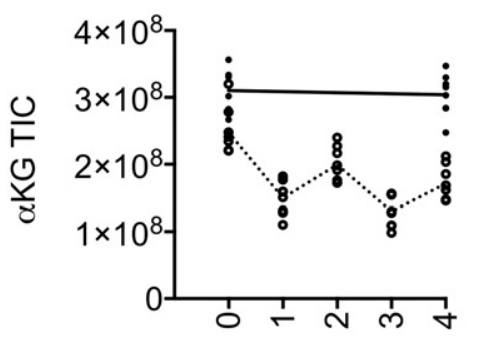

Cycles of Limb Ischemia

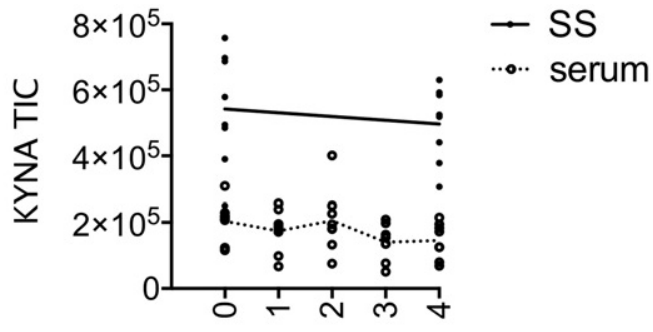

Cycles of Limb Ischemia

Figure 7. The behavior of serum (vena cava, VC) and sagittal sinus (SS) levels of aKG and KYNA during ischemia-reperfusion cycles in the RIPC group. aKG: alphaketoglutarate; KYNA: kynurenic acid; RIPC: remote ischemic preconditioning; SS: sagittal sinus; TIC: total ion chromatogram.

No significant change in $\alpha \mathrm{KG}$ were seen in the sagittal sinus samples following RIPC. KYNA levels were variable among animals, and levels did not change significantly in either the sagittal sinus or vena cava.

\section{Histopathology and immunohistochemistry}

The total histopathological sum score of CNS did not differ between the study groups $(\mathrm{p}=.854)$. The most defining finding was edema in both study groups $(\mathrm{p}=.764)$. Haemorrhages were observed only in the control group, but this difference did not reach a statistically significant difference between the groups $(\mathrm{p}=.317)$. Neuronal damage score did not differ between groups $(\mathrm{p}=.890)$. There were no infarction areas detected in the study groups.

In CNS, the total immunohistochemical sum scores of caspase $3(\mathrm{p}=.622)$ or $\mathrm{Nrf2}(\mathrm{p}=.583)$ did not differ between the study groups. The regional differences of the cytoplasmic Nrf2 scores were detected in the hippocampus and the cerebellum. In the cerebellum, the cytoplasmic Nrf2 scores were near-significantly higher in the RIPC group (Mean: 1.1, IQR: 0.0-2.5) compared with the control group (Mean: 0, IQR: 0.0-0.0), $(\mathrm{p}=.064)$ (Tables 1A and 1B).

\section{Discussion}

Postoperative mortality and neurological deficit remain the most serious complications related to the surgical repair of complex aortic and cardiac conditions. The operative technique of DHCA offers brain protective action, though further adjunctive methods are still required [2].
Clinically relevant experimental models match to this demand to develop protective strategies and assess their effectiveness and underlying mechanisms.

Encouraging experimental results concerning RIPC as a protective method reducing ischemia-reperfusion injury have been introduced to clinical settings in past years. However, in cardiac surgery, recently published results of two large, multicentre, randomized, controlled trials, ERICCA and RIPheart, failed to underline these previous results, indicating that the detection of the underlying protective mechanism requires further actions and understanding together with the considerations of the suitable patients and clinical settings to be utilised $[13,14]$.

In this study, the functional effect of RIPC was detected as a better cardiac index after weaning from the CPB in the intervention group (Figure 2(B)). The surgical setting itself, including manipulation of the heart and DHCA, explains the release of the cardiac enzymes in both groups. The lower lactate levels in the RIPC group indicate better surveillance under hypoxic conditions and indirectly better cellular and mitochondrial function (Figure 2(A)). Furthermore, better neurological outcome is associated with lower lactate levels [31]. Additionally, the serum levels of S100B protein declined faster to the baseline in the RIPC group, suggesting better tolerance against ischemic insult of DHCA (Figure 3) $[10,32]$. The possibility of extracerebral release of S100B needs to be taken into account $[33,34]$. However, the surgical trauma was identical for both study groups, as well as cardiotomy suction blood was not used targeting the release of S100B from the cerebral injury in the serum analysis [34].

During ischemia, Nrf2 expression is associated with neuronal cell protection [35]. Cytoplasmic Nrf2 staining reaction 
Table 1A. Immunohistochemistry.

\begin{tabular}{|c|c|c|c|c|c|c|c|}
\hline Protocol & Immunostaining & $\begin{array}{l}\text { Cortex } \\
\text { Score }\end{array}$ & $\begin{array}{c}\text { Thalamus } \\
\text { Score }\end{array}$ & $\begin{array}{c}\text { Hippocampus } \\
\text { Score }\end{array}$ & $\begin{array}{l}\text { Brainstem } \\
\text { Score }\end{array}$ & $\begin{array}{c}\text { Cerebellum } \\
\text { Score }\end{array}$ & $\begin{array}{l}\text { Total } \\
\text { Score }\end{array}$ \\
\hline RIPC mean & $\begin{array}{l}\text { Caspase } 3 \\
\text { (Nucleus) }\end{array}$ & $5.6(5.3-6.0)$ & $4.4(4.0-5.0)$ & $5.5(5.0-6.0)$ & $5.4(5.0-6.0)$ & $4.6(4.0-5.0)$ & $25.5(24.3-27.0)$ \\
\hline Control mean & & $5.6(5.3-6.0)$ & $4.3(3.5-5.0)$ & $5.6(5.5-6.0)$ & $4.9(4.0-6.0)$ & $3.9(3.5-4.5)$ & $25.0(23.0-26.0)$ \\
\hline p-value & & .727 & .883 & .593 & .281 & .138 & .622 \\
\hline RIPC mean & $\begin{array}{l}\text { Nrf2 } \\
\quad \text { (Cytoplasm) }\end{array}$ & $0.6(0.0-1.0)$ & $0.0(0.0-0.0)$ & $3.4(1.5-5.0)$ & $1.9(0.0-4.5)$ & $1.1(0.0-2.5)$ & $6.9(4.5-9.3)$ \\
\hline Control mean & & $0.6(0.0-1.0)$ & $1.0(0.0-2.0)$ & $5.1(4.5-6.0)$ & $1.0(0.0-2.0)$ & $0.0(0.0-0.0)$ & $8.1(5.0-10.0)$ \\
\hline$p$-value & & 1.000 & .143 & .084 & .442 & .064 & .583 \\
\hline
\end{tabular}

RIPC $n=8$, Control $n=8$. Immunohistochemical data after the 8-hour follow-up. Score; mean (IQR; 25th and 75th percentiles).

IQR: interquartile range; Nrf2: nuclear factor erythroid 2-related factor 2; RIPC: remote ischemic preconditioning.

Table 1B. Immunohistochemistry.

\begin{tabular}{|c|c|c|c|c|}
\hline Protocol & Immunostaining & $\begin{array}{c}\text { Kidney (glomerulus) } \\
\text { Score }\end{array}$ & $\begin{array}{c}\text { Kidney (tubular cells) } \\
\text { Score }\end{array}$ & $\begin{array}{l}\text { Heart } \\
\text { Score }\end{array}$ \\
\hline RIPC mean & $\begin{array}{l}\text { Caspase } 3 \\
\text { (Nucleus) }\end{array}$ & $5.8(6.0-6.0)$ & $6.0(6.0-6.0)$ & $5.8(5.5-6.0)$ \\
\hline $\begin{array}{l}\text { Control mean } \\
p \text {-value }\end{array}$ & & $\begin{array}{l}5.1(4.0-6.0) \\
.239\end{array}$ & $\begin{array}{l}6.0(6.0-6.0) \\
1.000\end{array}$ & $\begin{array}{l}5.8(5.5-6.0) \\
1.000\end{array}$ \\
\hline RIPC mean & $\begin{array}{l}\text { Nrf2 } \\
\quad \text { (Cytoplasm) }\end{array}$ & $2.9(0.0-5.0)$ & $3.6(2.0-5.0)$ & $4.0(3.5-4.5)$ \\
\hline $\begin{array}{l}\text { Control mean } \\
\text { p-value }\end{array}$ & & $\begin{array}{l}2.4(0.0-5.0) \\
.777\end{array}$ & $\begin{array}{l}2.0(0.0-5.0) \\
.358\end{array}$ & $\begin{array}{l}3.9(1.5-6.0) \\
.592\end{array}$ \\
\hline
\end{tabular}

RIPC $n=8$, Control $n=8$. Immunohistochemical data after the 8-hour follow-up. Score; mean (IQR; 25th and 75th percentiles).

IQR: interquartile range; Nrf2: nuclear factor erythroid 2-related factor 2; RIPC: remote ischemic preconditioning.

was in favour of the RIPC group in the cerebellum, indicating better neuronal cell protection against oxidative stress (Table 1(A)). Expression of caspase 3 is associated with the induction of DNA fragmentation and the activation of apoptosis [36]. Different grades of apoptotic events occurred in both study groups according to the caspase 3 findings. Limitations of this study, including a short follow-up period and a small group size, can partly explain the modest results of hematoxylin-eosin stainings.

Levels of $\alpha \mathrm{KG}$ decline in vena cava samples following RIPC, opposite to the accumulation seen following pharmacologic or genetic inhibition of EGLN1 in mice [28]. The activity of HIF prolyl 4-hydroxylases would be anticipated to decrease during ischemia and then increase during reperfusion in our ischemia-reperfusion model, which would not be the case following gene deletion or pharmacologic inhibitor studies. In support of this, we were unable to detect HIF1 $\alpha$ protein stabilization in muscle extracts (data not shown). It is possible that oscillations of $\alpha \mathrm{KG}$ levels seen in our study reflect repeated changes in EGLN1 activity, but further work would be needed to test this hypothesis. Additionally, no change in KYNA levels were seen in our study. Differences in model organism or differences between pseudohypoxia and ischemia likely contribute to this difference in KYNA and $\alpha \mathrm{KG}$ levels between our studies and prior work [28].

The proteomics analyses on sera from vena cava and sagittal sinus at two different time points of DHCA surgery althogether displayed 18 known porcine proteins, which appeared differentially expressed in response to the RIPC protocol compared with baseline and/or control values (Figures 4 and 5). All identified known porcine proteins were blood "residents" [37]. In addition to common blood circulating proteins (APOC3, APOE, HBB), the identified proteins also included coagulation or complement factors (F5, C3, C7), transport proteins (HBA, TTR, CBG) and other secreted and extracellular proteins (VTN, FGB, FBLN1, LAC, PGLYRP2, PC, IGFALS, A1BG). The identified proteins play a role in a range of biological processes (PANTHER) (Figure 5), suggesting that the RIPC-mediated neuroprotective mechanism(s) during DHCA in porcine model probably involve complex synergy between different signaling pathways.

Specific attention was given to porcine proteins putatively relevant for RIPC protection (19-20, 314), namely APOE, C3, TTR, and VTN, in the sagittal sinus blood samples. Our results showed, however, no significant changes in their concentration between the RIPC and control group at any time point (Figure 6), suggesting that those proteins were likely not involved in mediating the protective effects of RIPC in that particular time-frame of DHCA surgical protocol. Due to the limited number of annotated porcine proteins in the public databases, identification of $>50 \%$ of the detected proteins was impossible. Therefore, we cannot exclude the possibility that these so far uncharacterized proteins contributed to the remote ischemic preconditioning.

\section{Conclusions}

The faster recovery of serum S100B levels suggests better ischemic tolerance by the brain of RIPC. The lower systemic lactate suggests better cellular and mitochondrial function after global ischemia in the RIPC group. The higher regional cytoplasmic Nrf2 scores indicate possible favourable neuronal cell protection against oxidative stress in the RIPC group. Similarly, the better cardiac index signifies functional effects of RIPC postoperatively. 
We were not able to identify any specific protein mediating the effect of RIPC. However, the RIPC-induced effectors do not necessarily have to be proteins. They could be circulating metabolites, non-peptide hormones or combinations of several factors. Therefore, additional and more analytically deepened research needs to be carried out to identify global changes in peptides, non-peptide hormones, and other metabolites following the RIPC protocol.

\section{Acknowledgements}

The authors wish to thank Pasi Ohtonen, MSc, for his assistance with the statistical analysis and Seija Seljänperä, RN, and Tanja Aatsinki, TA, for their excellent technical assistance.

\section{Disclosure statement}

Dr. Tatu Juvonen is principal investigator in a study on central nervous system protection in cardiac and aortic surgery at the Research Unit of Surgery, Anesthesia and Intensive care, University of Oulu and MRC Oulu. Dr. Peppi Koivunen is professor and principal investigator at Biocenter Oulu and Faculty of Biochemistry and Molecular Medicine (FBMM), University of Oulu. Dr. Benjamin Olenchock acknowledges research support from the National Institutes of Health (NIH) and Gilead Sciences, Inc.

The authors report no conflicts of interest.

\section{Funding}

This work was supported by the The Finnish Foundation for Cardiovascular Research, The Sigrid Jusélius Foundation, Oulu University Hospital (Professor Tatu Juvonen). The Academy of Finland Grants 218129, The Sigrid Jusélius Foundation, The Emil Aaltonen Foundation, The Jane and Aatos Erkko Foundation (Professor Peppi Karppinen). National Institutes of Health (NIH), Gilead Sciences, Inc (MD, PhD Benjamin Olenchock).

\section{References}

[1] Kouchoukos NT, Kulik A, Castner CF. Outcomes after thoracoabdominal aortic aneurysm repair using hypothermic circulatory arrest. J Thorac Cardiovasc Surg. 2013;145(3 Suppl): S139-S141.

[2] Ziganshin BA, Rajbanshi BG, Tranquilli M, et al. Straight deep hypothermic circulatory arrest for cerebral protection during aortic arch surgery: safe and effective. J Thorac Cardiovasc Surg. 2014;148:888-98; discussion 898-900.

[3] Kouchoukos NT, Scharff JR, Castner CF. Repair of primary or complicated aortic coarctation in the adult with cardiopulmonary bypass and hypothermic circulatory arrest. J Thorac Cardiovasc Surg. 2015;149:S83-S85.

[4] Erecinska M, Thoresen M, Silver IA. Effects of hypothermia on energy metabolism in mammalian central nervous system. J Cereb Blood Flow Metab. 2003;23:513-530.

[5] González-Ibarra FP, Varon J, Lopez-Meza EG. Therapeutic hypothermia: critical review of the molecular mechanisms of action. Front Neur. 2011;2:4.

[6] Gega A, Rizzo JA, Johnson MH, et al. Straight deep hypothermic arrest: experience in 394 patients supports its effectiveness as a sole means of brain preservation. Ann Thorac Surg. 2007;84:759-766. discussion 766-767.

[7] Murry CE, Jennings RB, Reimer KA. Preconditioning with ischemia: a delay of lethal cell injury in ischemic myocardium. Circulation. 1986;74:1124-1136.
[8] Przyklenk K, Bauer B, Ovize M, et al. Regional ischemic 'preconditioning' protects remote virgin myocardium from subsequent sustained coronary occlusion. Circulation. 1993;87:893-899.

[9] Gho BC, Schoemaker RG, van den Doel MA, et al. Myocardial protection by brief ischemia in noncardiac tissue. Circulation. 1996;94:2193-2200.

[10] Jensen H, Loukogeorgakis S, Yannopoulos F, et al. Remote ischemic preconditioning protects the brain against injury following hypothermic circulatory arrest. Circulation. 2011;123:714-721.

[11] Ali ZA, Callaghan CJ, Lim E, et al. Remote ischemic preconditioning reduces myocardial and renal injury after elective abdominal aortic aneurysm repair: a randomized controlled trial. Circulation. 2007;116:98-105.

[12] Thielmann M, Kottenberg E, Kleinbongard P, et al. Cardioprotective and prognostic effects of remote ischaemic preconditioning in patients undergoing coronary artery bypass surgery: a single-centre randomised, double-blind, controlled trial. Lancet. 2013;382:597-604.

[13] Hausenloy DJ, Candilio L, Evans R, ERICCA Trial Investigators, et al. Remote ischemic preconditioning and outcomes of cardiac surgery. N Engl J Med. 2015;373:1408-1417.

[14] Meybohm P, Bein B, Brosteanu O, for the RIPHeart Study Collaborators*, et al. A multicenter trial of remote ischemic preconditioning for heart surgery. $\mathrm{N}$ Engl J Med. 2015;373:1397-1407.

[15] Gill R, Kuriakose R, Gertz ZM, et al. Remote ischemic preconditioning for myocardial protection: update on mechanisms and clinical relevance. Mol Cell Biochem. 2015;402:41-49.

[16] Konstantinov IE, Li J, Cheung MM, et al. Remote ischemic preconditioning of the recipient reduces myocardial ischemia-reperfusion injury of the denervated donor heart via a Katp channel-dependent mechanism. Transplantation. 2005;79:1691-1695.

[17] Shimizu M, Tropak M, Diaz RJ, et al. Transient limb ischaemia remotely preconditions through a humoral mechanism acting directly on the myocardium: evidence suggesting cross-species protection. Clin Sci. 2009;117:191-200.

[18] Michelsen MM, Stottrup NB, Schmidt MR, et al. Exerciseinduced cardioprotection is mediated by a bloodborne, transferable factor. Basic Res Cardiol. 2012;107:260.

[19] Hepponstall M, Ignjatovic V, Binos S, et al. Remote ischemic preconditioning (RIPC) modifies plasma proteome in humans. PLoS One. 2012;7:e48284.

[20] Hibert P, Prunier-Mirebeau D, Beseme O, et al. Apolipoprotein a-I is a potential mediator of remote ischemic preconditioning. PLoS One. 2013;8:e77211.

[21] Hibert P, Prunier-Mirebeau D, Beseme O, et al. Modifications in rat plasma proteome after remote ischemic preconditioning (RIPC) stimulus: identification by a SELDI-TOF-MS approach. PLoS ONE. 2014;9:e85669.

[22] Chao de la Barca JM, Bakhta O, Kalakech H, et al. Metabolic signature of remote ischemic preconditioning involving a cocktail of amino acids and biogenic amines. J Am Heart Assoc. 2016;5:e003891.

[23] Dickson EW, Lorbar M, Porcaro WA, et al. Rabbit heart can be "preconditioned" via transfer of coronary effluent. Am J Physiol. 1999;277:H2451-H2457.

[24] Skyschally A, Gent S, Amanakis G, et al. Across-species transfer of protection by remote ischemic preconditioning with speciesspecific myocardial signal transduction by reperfusion injury salvage kinase and survival activating factor enhancement pathways. Circ Res. 2015;117:279-288.

[25] Breivik L, Helgeland E, Aarnes EK, et al. Remote postconditioning by humoral factors in effluent from ischemic preconditioned rat hearts is mediated via PI3K/Akt-dependent 
cell-survival signaling at reperfusion. Basic Res Cardiol. 2011;106:135-145.

[26] Bell KF, Fowler JH, Al-Mubarak B, et al. Activation of Nrf2regulated glutathione pathway genes by ischemic preconditioning. Oxid Med Cell Longev. 2011;2011:689524.

[27] Itoh K, Mimura J, Yamamoto M. Discovery of the negative regulator of Nrf2, Keap1: a historical overview. Antioxid Redox Signal. 2010;13:1665-1678.

[28] Olenchock BA, Moslehi J, Baik AH, et al. EGLN1 inhibition and rerouting of $\alpha$-ketoglutarate suffice for remote ischemic protection. Cell. 2016;164:884-895.

[29] Meller R, Simon RP. A critical review of mechanisms regulating remote ischemic preconditioning-induced brain protection. J Appl Physiol. 2015;119:1135-1142.

[30] Pang T, Zhao Y, Zhang NR, et al. Transient limb ischemia alters serum protein expression in healthy volunteers: complement $\mathrm{C} 3$ and vitronectin may be involved in organ protection induced by remote ischemic preconditioning. Oxid Med Cell Longev. 2013;2013:859056.

[31] Lee DH, Cho IS, Lee SH, Korean Hypothermia Network Investigators, et al. Correlation between initial serum levels of lactate after return of spontaneous circulation and survival and neurological outcomes in patients who undergo therapeutic hypothermia after cardiac arrest. Resuscitation. 2015;88:143-149.

[32] Yokobori S, Hosein K, Burks S, et al. Biomarkers for the clinical differential diagnosis in traumatic brain injury-a systematic review. CNS Neurosci Ther. 2013;19:556-565.

[33] Anderson RE, Hansson L-O, Nilsson O, et al. High serum S100B levels for trauma patients without head injuries. Neurosurgery. 2001;48:1255-1260.

[34] Anderson RE, Hansson L-O, Nilsson O, et al. Increase in serum S100A1-B and S100BB during cardiac surgery arises from extracerebral sources. Ann Thorac Surg. 2001;71:1512-1517.

[35] Shih AY, Li P, Murphy TH. A small-molecule-inducible Nrf2mediated antioxidant response provides effective prophylaxis against cerebral ischemia in vivo. J Neurosci. 2005;25:10321-10335.

[36] Sakurai M, Nagata T, Abe K, et al. Survival and death-promoting events after transient spinal cord ischemia in rabbits: induction of Akt and caspase3 in motor neurons. J Thorac Cardiovasc Surg. 2003;125:370-377.

[37] Zhang K, Liu Y, Shang Y, et al. Analysis of pig serum proteins based on shotgun liquid chromatography-tandem mass spectrometry. Afr J Biotechnol. 2012;11:12118-12127. 